\title{
Accuracy of acetabular cup positioning using imageless navigation
}

Erik Hohmann ${ }^{1,2^{*}}$, Adam Bryant ${ }^{3}$ and Kevin Tetsworth ${ }^{4,5}$

\begin{abstract}
Background: Correct placement of the acetabular cup is a crucial step in total hip replacement to achieve a satisfactory result and remains a challenge with free-hand techniques. Imageless navigation may provide a viable alternative to free-hand technique and improve placement significantly. The purpose of this project was to assess and validate intra-operative placement values for both inclination and anteversion as displayed by an imageless navigation system to post-operative measurement of cup position using high resolution CT scans.
\end{abstract}

Methods: Thirty-two subjects who underwent primary hip joint arthroplasty using imageless navigation were included. The average age was 66.5 years (range 32-87). 23 non-cemented and 9 cemented acetabular cups were implanted. The desired position for the cup was 45 degrees of inversion and 15 degrees of anteversion. A pelvic CT scan using a multi-slice CT was used to assess the position of the cup radiographically.

Results: Two subjects were excluded because of dislodgement of the tracking pin. Pearson correlation revealed a strong and significant correlation $(r=0.68 ; p<0.006)$ for cup inclination and a moderate non-significant correlation $(r=0.53 ; p=0.45)$ between intra-operative readings and cup placement for anteversion.

Conclusions: These findings can be explained with the possible introduction of systematic error. Even though the acquisition of anatomic landmarks is simple, they must be acquired with great precision. An error of $1 \mathrm{~cm}$ can result in a mean anteversion error of 6 degrees and inclination error of 2.5 degrees. Whilst computer assisted surgery results in highly accurate cup placements for inclination, anteversion of the cup cannot be determined accurately.

\section{Background}

Correct placement of the acetabular cup in total hip arthroplasty is a crucial step to achieve a satisfactory result and remains a challenge with free-hand techniques [1-3]. Indeed, malpositioning can induce early loosening, high wear and postoperative dislocation [4-6]. Various investigators have demonstrated that conventional free-hand positioning can result in a high percentage of unacceptable acetabular cup placements $[2,3,7,8]$.

Imageless navigation may provide a viable alternative to free-hand techniques and the use of mechanical guides and may improve placement significantly $[7,9,10]$. Previous authors have demonstrated that cup alignment significantly improved with the use of computer navigation [3,9,11-14]. Imageless computer aided navigation

\footnotetext{
* Correspondence: ehohmann@optusnet.com.au

'Musculoskeletal Research Unit, CQ University, Yaamba Road, Rockhampton 4700, Australia

Full list of author information is available at the end of the article
}

relies on a pelvic coordinate system which uses bony landmarks (anterior superior iliac spines and pubic tubercle) to define the anterior frontal plane $[15,16]$. These bony landmarks are determined by palpation and digitization through the overlying soft-tissue with a metal pointer [16]. Manual digitization can potentially cause measurement error which, in turn, can result in excessive tilt of the cup in the frontal plane. This is particularly problematic in obese patients where excess soft tissue can completely obscure bony landmarks. Clearly, the introduction of systematic error may lead to cup placement which differs from the intra-operative readings when using imageless navigation.

Therefore, the purpose of this study was to assess and validate intra-operative placement orientation as displayed by the navigation unit to post-operative measurement of cup position using high resolution CT scans. We hypothesized that inclination is highly accurate as the anterior superior iliac spines are easily palpable even

\section{Biomed Central}


in obese patients. In contrast, we hypothesized that anteversion is inaccurate due to the underlying soft-tissue and the difficulty in identifying the pubic tubercle.

\section{Methods}

\section{Patient selection}

Between June 2005 and December 2007, 32 patients underwent primary hip joint replacement using imageless navigation. Two patients had to be excluded because of intra-operative dislodgement of the tracking pin. The mean patient age was $66.5 \pm 14$ (range 28-87) years. There were 16 males (mean age $62.2 \pm 12.2$ ) and 14 females (mean age $71.4 \pm 14.7$. The mean weight was $85.6 \pm 14 \mathrm{~kg}$ (range 57-112), the mean height measured $169 \pm 8.6 \mathrm{~cm}$ (range153-186) and the average BMI was $30.04 \pm 4.6 \mathrm{~kg} / \mathrm{m}^{2}$ (range 20.9-39.5). Twenty one noncemented and 9 cemented acetabular cups were implanted. The average size of the non-cemented cup was $53 \mathrm{~mm}$ (range 46-60) and averaged 54 (range 5058 ) for the cemented cup. The main indication was primary osteoarthritis $(n=25)$, osteonecrosis $(n=5)$, displaced neck of femur fracture $(n=1)$ and failed screw fixation with head collapse after neck of femur fracture $(n=1)$. In 17 subjects, hip arthroplasty was performed on the right hip and 15 subjects had a left total hip arthroplasty. Surgery was performed by a single surgeon who was an experienced user of the imageless navigation system.

\section{Sequence of Navigation}

An imageless computer navigation system (Stryker ${ }^{\circledR}$ Navigation System, Stryker Corporation, Kalamazoo, MI, USA) was used for all surgery. Patients were placed supine. A Schanz screw was inserted into the ipsilateral anterior superior iliac spine (ASIS) through a stab incision. The pelvic navigation tracker was attached to the screw. Bony landmarks (ASIS, pubic tubercle) were determined and digitalized with a metal pointer (Figure 1). Once the frontal plane was defined by the computer the hip was moved through arrange of motion to determine the centre of rotation. Prior to dislocation and resection of the femoral head the piriformis fossa was digitalized. The acetabular fossa and rim was then digitalized. Once the landmarks were defined, the navigation system determined inclination and anteversion of the acetabulum.

\section{Surgical Technique}

The surgical procedures were performed using a lateral Hardinge approach in all cases. Reaming, trial cup position and final cup position was performed navigated. The aim was to achieve 45 degrees inclination and 15 degrees anteversion. Intra-operative cup position was recorded. The contemporary cup $\left(\right.$ Stryker $\left.^{\circledR}\right)$ was used

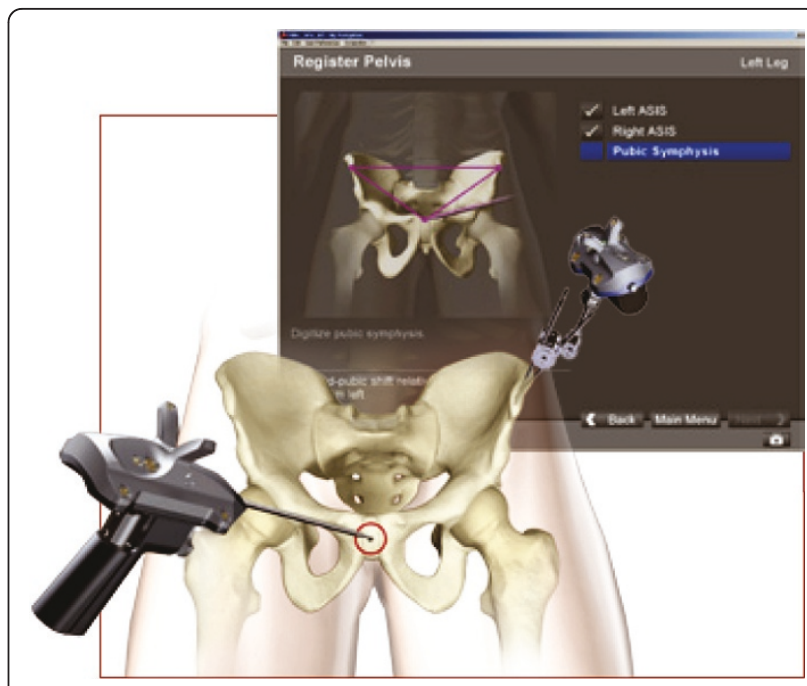

Figure 1 Once the Schanz screw was inserted into the ipsilateral anterior superior iliac, spine and the pelvic navigation tracker was attached, bony landmarks (ASIS, pubic, tubercle) were determined and digitalized with a metal pointer.

with cement and the Trident cup $\left(\right.$ Stryker $\left.^{\circledR}\right)$ was used without cement.

\section{Postoperative CT}

Post-operatively, a multi-slice CT scan was obtained on day one post surgery using a helical CT scanner (Somatom; Siemens ${ }^{\circledR}$, Munich, Germany). All CT scans were performed by the same radiology technician to a preestablished protocol. Two millimeter slices were obtained in all cases. The position of the pelvis was standardized by reformatting the images to the frontal plane defined by both anterior superior iliac spines and the pubic tubercle. The largest cup diameter on the coronal plane was identified and the inclination was measured. Similar anteversion was measured by identifying the largest cup diameter on an axial plane. All measurements were performed three times and averaged.

\section{Statistical Analysis}

To determine sample size a power calculation was performed. The study was designed to provide the number of cases required to discover a statistical significant $(p=0.05)$ correlation of $r \geq 0.50$ between intra-operative cup placement and post-operative CT measurements. The sample size calculation based on these parameters indicated that 29 patients were needed to provide $90 \%$ statistical power.

Pearson's product-moment correlation coefficients were used to establish the strength of the relationships between intra-operative cup placement and post-operative CT measurements. All analyses were conducted using SPSS (Version 12.0.1; Chicago, IL) for Windows. 


\section{Results}

\section{Inclination}

Differences between navigation-derived intra-operative final cup inclination and final CT cup inclination of all 30 cups are shown in Figure 2. In 23 subjects, cup placement was within 5 degrees of intra-operative readings. Six cups were placed within 10 degrees and one cup was placed with a difference of more than 10 degrees (Figure 2). A mean difference of $3.8^{0}+3.8^{0}$ (range $0^{\circ}$ $15.7^{\circ}$ ) between intra-operative cup placement and postoperative measurement was observed. Pearson correlation revealed a strong, significant correlation $(\mathrm{r}=0.68 ; p$ $<0.006$ ) for cup inclination between intra-operative final cup placement and cup placement measured by CT.

\section{Anteversion}

Differences between navigation-derived intra-operative final cup anteversion and final CT cup anteversion of all 30 cups are shown in Figure 3. Only 11 cups were placed within 5 degrees of navigation unit readings. In 13 cups anteversion readings and final CT results were within 10 degrees and 6 cups were placed outside 10 degrees (Figure 3). A mean difference of $7.7^{0}+7.6^{0}$ (range $0^{\circ}-26^{\circ}$ ) between intra-operative cup placement and post-operative measurement was observed. Pearson correlation revealed a moderate, non-significant correlation $(\mathrm{r}=0.53 ; p=0.45)$ between intra-operative readings and cup placement.

\section{Discussion}

Imageless navigation is absolutely dependent upon accurate identification and digitization of appropriate bony landmarks. Unfortunately, anatomical landmarks are often obscured in larger patients, and may lead to corresponding difficulty in positioning an implant accurately. To assess the clinical viability of various landmarks and particular methods for imageless navigation, it is necessary to evaluate the accuracy of navigated cup position

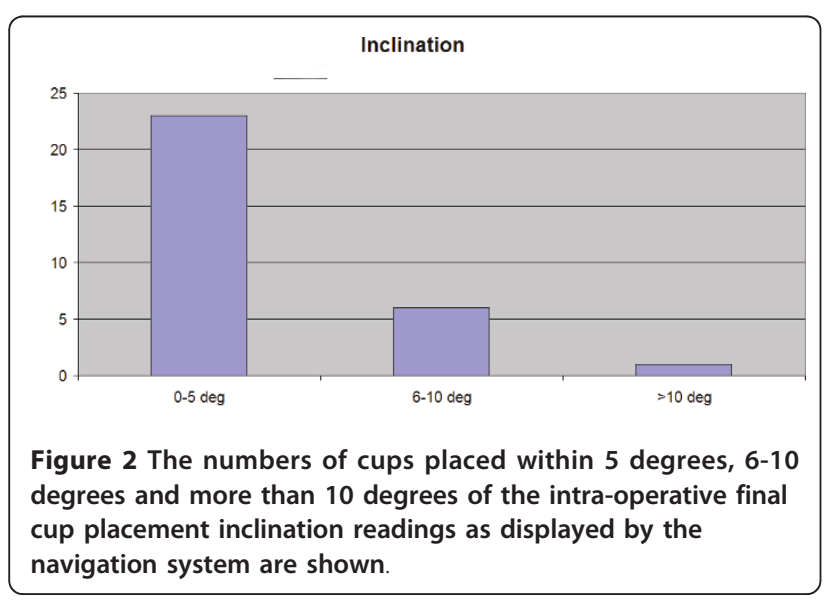

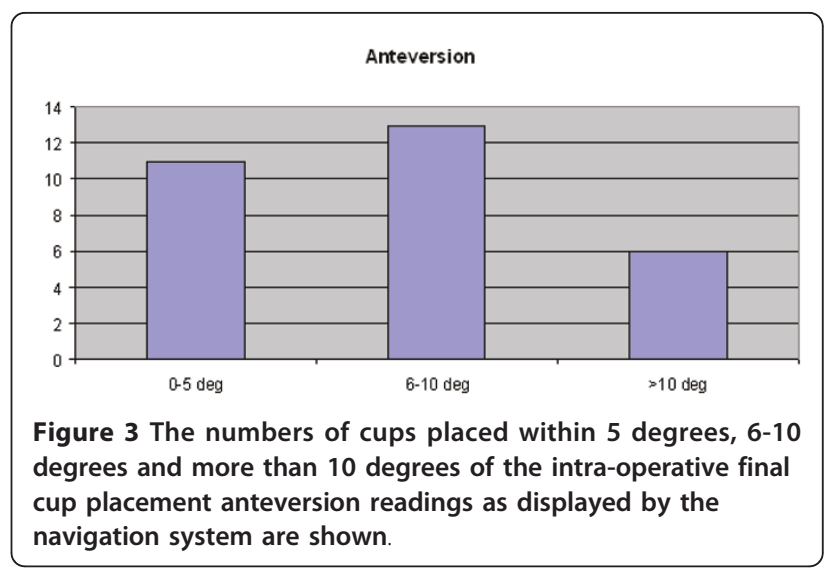

intra-operative values in comparison to the implants final position when measured objectively post-operatively. Using one specific technique for imageless navigation, our results demonstrate there was a strong $(\mathrm{r}=$ $0.68)$ and $(p=0.006)$ and significant relationship for inclination, but only a moderate non-significant relationship $(\mathrm{r}=0.36)$ for anteversion when comparing intraoperative cup position and post-operative final implant position measurements.

These results compare favorably with those previously published by other authors.

Ybinger et al [17] observed a mean difference between navigation recorded and $\mathrm{CT}$ measured inclination of $3.5^{\circ}$ degrees and a mean difference $6.5^{\circ}$ degrees for anteversion in 37 subjects. In an earlier laboratory study with 10 cadavers, Kalteis et al [18] observed a median difference of $1.5^{\circ}$ for inclination and $0.5^{\circ}$ for anteversion. Fukunishi et al [19] analyzed accuracy of cup navigation in 27 total hip arthroplasties. Intra-operative cup inclination ranged from $39.9^{\circ}$ to $46.6^{\circ}$ degrees with a mean angle of $43.5^{\circ}$ degrees compared to a range of $38.1^{\circ}$ to $55.0^{\circ}$ degrees with a mean angle of $44.9^{\circ}$ degrees post-operative. Mean intra- and post-operative values were $11.1^{\circ}$ (range $0-17.8$ ) degrees and $13.5^{\circ}$ (range 5.1-21.6) degrees respectively. A discrepancy of $>$ $5^{0}$ degrees was observed in one case. A mean difference of $1.9^{\circ}$ degrees for inclination and $2.6^{\circ}$ degrees for anteversion was calculated between intra- and post-operative values. Dorr et al [12] observed an accuracy of $4.4^{\circ}$ degrees for inclination and $4.1^{\circ}$ degrees for anteversion with no outliers greater than $5^{0}$ degrees. They concluded that surgeons can trust a validated computer navigation system for cup position.

Our results compare favorably with these previous studies, although only Fununkashi et al [19] (one patient with a discrepancy of $>5^{0}$ degrees) and Dorr et al [12] (no outliers) reported outliers. In contrast to the previous authors [12,17-19], we documented similar differences but we have observed more frequent cup 
placements with a discrepancy of $>5^{0}$ degrees. It may therefore be more important to report on the numbers of outliers rather than documenting mean differences, ranges and standard deviations. This would perhaps be a better method to describe the accuracy of a navigation system more definitely.

One possible explanation for the differences between our results and other authors may be attributed to the fact that that average BMI of our cohort group is above 30. In this respect, it has been demonstrated by several authors [20-22] that the overlying soft tissue obscures bony landmarks and introduces measurement error. Ybinger et al [17] reported a positive moderate, significant $(\mathrm{r}=0.44, p=0.007)$ relationship between thickness of soft tissues over the ASIS and inclination as well as positive moderate, significant correlation $(\mathrm{r}=0.52, p=$ 0.001 ) between soft tissues over the pubic tubercles and anteversion angles.

When digitizing the ASIS, measurement errors of one centimeter (left versus right) and two centimeters may introduce errors up to $2.5^{\circ}$ and $5^{\circ}$ degrees in cup alignment (Figure 4). Similarly, if digitization of the pubic symphysis is measured either one centimeter too anterior or posterior, a measurement error of $6^{\circ}$ will result. A difference of 2 centimeters increases the error to $11^{\circ}$ (Figure 5).

The difficulty in palpating the pubic symphysis in subjects with a thicker soft tissue envelope and the shorter distance between the superior aspect of the anterior pelvic triangle and the symphysis explains the higher error that we observed for anteversion. Consequently, obese patients may not be suitable for hip navigation given the

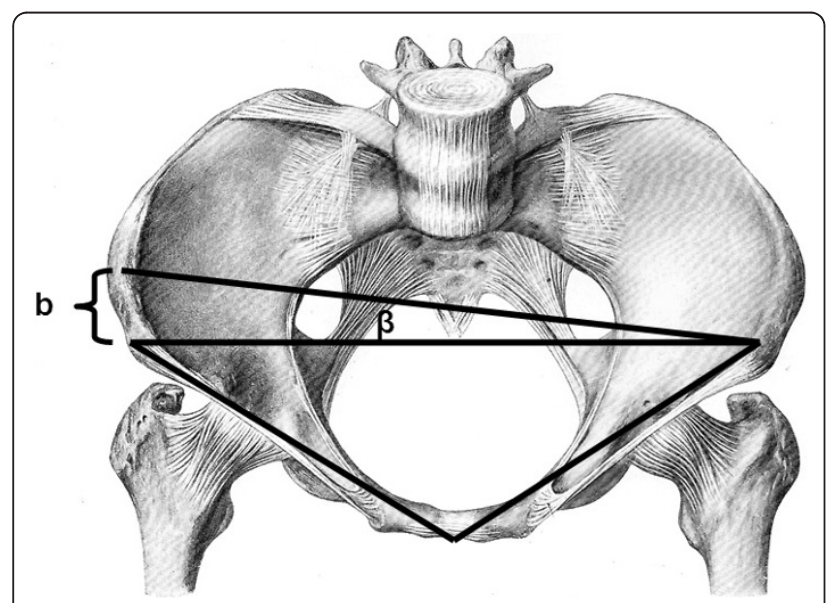

Figure 4 Failure to digitize the anterior iliac spines correctly can introduce systematic error. A difference of one centimeters ("b") between the right and left ASIS introduces an error of 2.5 and a difference of two centimeters ("b") can result in a 5 degree error for inclination.

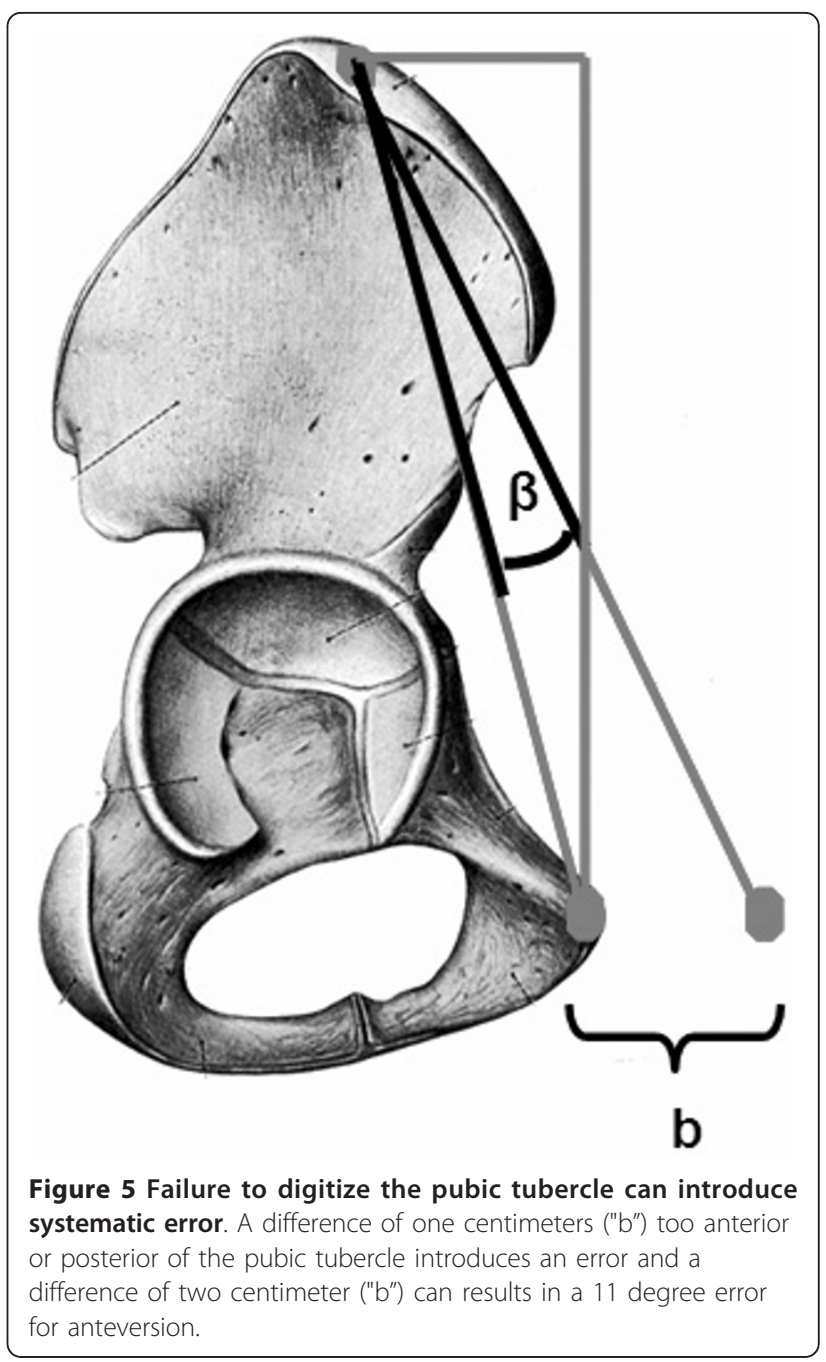

increased risk of measurement error. However this criteria was not studied in this project.

\section{Conclusion}

The results of our study suggest that there is a strong and significant correlation between intra-operative final cup placement and post-operative values for inclination and a moderate non-significant correlation for anteversion. Furthermore, we demonstrated cemented cup placement is more accurate, despite the relatively small sample size. Although the location of anatomic landmarks is simple; precision is imperative in order to reduce error. These findings are most likely due to the introduction of systematic error. Small acquisition errors can result in substantial systematic errors introduced by inadequate calculation of the anterior pelvic plane by the navigation system. The results of this study suggest that imageless navigation is a tool which is reliable, easy to use and potentially reduces the variation in free-hand placement of acetabular cups. Further work is warranted 
to increase the precision of cup positioning using this particular navigation system.

\section{Author details}

'Musculoskeletal Research Unit, CQ University, Yaamba Road, Rockhampton 4700, Australia. ${ }^{2}$ Department of Orthopaedic Surgery, Rockhampton Hospital, Canning Street, Rockhampton QLD 4700, Australia. ${ }^{3}$ Centre for Health, Exercise and Sports Medicine, Faculty of Medicine, The University of Melbourne, 200 Berkeley Street, Melbourne VIC 3010, Australia. ${ }^{4}$ Department of Orthopaedic Surgery, Royal Brisbane Hospital, Butterfield Street, Herston QLD 4029, Australia. ${ }^{5}$ CONROD Professor of Orthopaedic Trauma Surgery, Division of Surgery, University of Queensland Medical School, Butterfield Street, Herston QLD 4029.

\section{Authors' contributions}

EH: chief investigator, developed design and methods, analyzed data, drafted manuscript and is responsible for the final approval of the manuscript

AB:assisted with the design and analysis, assisted with the first draft and critically reviewed further versions, co-author who applied all statistical analysis and was involved in interpretation of results.

$\mathrm{KT}$ :assisted with the design and analysis, assisted with the first draft and critically reviewed further versions

\section{Competing interests}

The authors declare that they have no competing interests.

Received: 30 January 2011 Accepted: 10 August 2011

Published: 10 August 2011

\section{References}

1. Blendea S, Troccaz J, Ravey JN, Merloz P: Image-free cup navigation inaccuracy: a two-study approach. Computer Aided Surgery 2007, 12(3):176-180

2. Bosker BH, Verheyen CCPM, Horstman WG, Tulp NJA: Poor accuracy of freehand cup positioning during total hip arthroplasty. Arch Orthop Trauma Surg 2007, 127:375-379.

3. Saxler G, Marx A, Vandevelde D, Langlotz U, Tannast M, Wiese M, Michaelis U, Kemper G, Grützner PA, Steffen R, von Knoch M, HollandLetz T, Bernsmann K: The accuracy of free-hand cup positioning-a CT based measurement of cup placement in 105 total hip arthroplasties. Int Orthop 2004, 28(4):198-201.

4. Jenny JY, Boeri C, Dosch JC, Uscatu M, Ciobanu E: Navigated nom-image based positioning of the acetabulum during total hip replacement. Int Orthop 2009, 33:83-87.

5. Kennedy JG, Rogers WB, Soffe KE, Sullivan RJ, Griffen DG, Sheehan LJ: Effect of acetabular component orientation on recurrent dislocation, pelvic osteolysis, polyethylene wear, and component migration. J Arthroplasty 1998, 5:530-534.

6. Ryan JA, Jamali AA, Bargar WL: Accuracy of computer navigation for acetabular component placement in THA. Clin Orth Relat Res 2010, 468:169-177.

7. Digioia AM III, Jaramaz B, Plakseychuk AY, Moody JE Jr, Nikou C, Labarca RS, Levison TJ, Picard F: Comparison of a mechanical acetabular alignment guide with computer placement of the socket. J Arthroplasty 2002, 17(3):359-364

8. Honl M, Schwieger K, Salineros M, Jacobs J, Morlock M, Wimmer M: Orientation of the acetabular component. A comparison of five navigation systems with conventional surgical technique. J Bone Joint $\mathrm{Br}$ 2006, 88B:1401-1405.

9. Kalteis T, Handel M, Herold T, Perlick L, Baethis H, Grifka J: Greater accuracy in positioning of the acetabular cup by using an image-free navigation system. Int Orthop 2005, 29:272-276.

10. Jolles BM, Genoud P, Hoffmeyer P: Computer-assisted placement in total hip arthroplasty improve accuracy of placement. Clin Orth Relat Res 2004, 426:174-179.

11. Haaker RG, Tiedjen K, Ottersbach A, Rubenthaler F, Stockheim M, Stiehl JB: Comparison of conventional versus computer-navigated acetular component insertion. J Arthroplasty 2007, 22(2):151-159.
12. Dorr LD, Malik A, Wan Z, Long WT, Harris M: Precision and bias of imageless computer navigation and surgeon estimates for acetabular component position. Clin Orth Relat Res 2007, 465:92-99.

13. Kalteis T, Handel M, Bathis H, Perlick L, Tingart M, Grifka J: Imageless navigation for insertion of the acetabular component in total hip arthroplasty: is it as accurate as CT-based navigation? I Bone Joint Surg Br 2006, 88:163-167.

14. Parratte $\mathrm{S}$, Argenson JN: Validation and usefulness of a computer-assisted cup-positioning system in total hip arthroplasty. A prospective, randomized, controlled study. J Bone Joint Surg Am 2007, 89:494-499.

15. Lin F, Lim D, Wixon RL, Milos S, Hendrix RW: Validation of a computer navigation system and a CT method for determination of the orientation of implanted acetabular cup in total hip arthroplasty: a cadaver study. Clinical Biomechanics 2008, 23:1004-1011.

16. Nogler M, Kessler O, Prass LA, Donnelly B, Streicher R, Sledge J: Reduced variability of acetabular cup positioning with use of an imageless navigation system. Clin Orthop Relat Res 2004, 426:159-163.

17. Ybinger T, Kumpan W, Hoffart HE, Muschalik B, Bullmann W, Zweymüller K Accuracy of navigation-assisted acetabular component positioning studied by computed tomography measurements. J Arthroplasty 2007 22(6):812-817.

18. Kalteis T, Beckmann J, Herold T, Zysk S, Bathis H, Perlick L, Grifka J: Genauigkeit eines bildfreien Navigationssystems für die Hüftpfannenimplantation-eine anatomische Studie. Biomed Technik 2004 49:257-262.

19. Fukunishi S, Fukui T, Imamura F, Nishio S: Assessment of accuracy of acetabular cup orientation in CT-free navigated total hip arthroplasty. Orthopedics 2008, 31:10.

20. Spencer JMF, Day RE, Sloan KE, Beaver RJ: Computer navigation of the acetabular component. A cadaver reliability study. J Bone Joint Br 2006, 88b:972-975.

21. Wolf A, DiGioia AM III, Mor AB, Jaramaz B: Cup alignment error model for total hip arthroplasty. Clin Orthop Relat Res 2005, 437:132-137.

22. Parratte $\mathrm{S}$, Argenson JN: Validation and usefulness of a computer-assisted cup-positioning system in total hip arthroplasty. A prospective, randomized, controlled study. J Bone Joint Surg Am 2007, 89:494-499.

doi:10.1186/1749-799X-6-40

Cite this article as: Hohmann et al: Accuracy of acetabular cup positioning using imageless navigation. Journal of Orthopaedic Surgery and Research 2011 6:40

\section{Submit your next manuscript to BioMed Central and take full advantage of:}

- Convenient online submission

- Thorough peer review

- No space constraints or color figure charges

- Immediate publication on acceptance

- Inclusion in PubMed, CAS, Scopus and Google Scholar

- Research which is freely available for redistribution 